\title{
Tissue-wide integration of mechanical cues promotes effective auxin patterning
}

\author{
João R. D. Ramos ${ }^{1}$, Alexis Maizel $^{2}$ (D) Karen Alim $^{1,3, a}{ }_{\mathbb{D}}$ \\ ${ }_{1}^{1}$ Max Planck Institute for Dynamics and Self-Organization, Göttingen, Germany \\ 2 Center for Organismal Studies, University of Heidelberg, Heidelberg, Germany \\ 3 Physik-Department, Technische Universität München, Munich, Germany
}

Received: 30 September 2020 / Accepted: 7 February 2021

(C) The Author(s) 2021

\begin{abstract}
New plant organs form by local accumulation of auxin, which is transported by PIN proteins that localize following mechanical stresses. As auxin itself modifies tissue mechanics, a feedback loop between tissue mechanics and auxin patterning unfolds-yet the impact of tissue-wide mechanical coupling on auxin pattern emergence remains unclear. Here, we use a model composed of a vertex model for plant tissue mechanics and a compartment model for auxin transport to explore the collective mechanical response of the tissue to auxin patterns and how it feeds back onto auxin transport. We compare a model accounting for a tissue-wide mechanical integration to a model that regards cells as mechanically isolated. We show that tissue-wide mechanical coupling not only leads to more focused auxin spots via stress redistribution, but that it also mitigates the disruption to patterning when considering noise in the mechanical properties of each cell of the tissue. We find that this mechanism predicts that a local turgor increase correlates with auxin concentration, and yet auxin spots can exist regardless of the exact local turgor distribution.
\end{abstract}

\section{Introduction}

Formation of organs entails an effective coordination of local cell growth typically initiated by patterns of one or more morphogenic factors. Understanding how these patterns of morphogenic agents robustly emerge is fundamental for predicting organ morphogenesis. Plants organ formation is interesting from a physical perspective due to the strong mechanical coupling between plant cells, and the fact that growth is driven by changes in the mechanical properties of the cell wall and internal pressure [1-5]. Evidence indicates that the morphogenic factors such as the plant hormone auxin change the mechanics of the tissue [6,7], with implications for the shaping of organs $[8,9]$. Interestingly, the transporters of auxin respond to mechanical cues $[10,11]$, leading to an intertwining of chemical and mechanical cues.

The phytohormone auxin, Indole-3-Acetic Acid, is the key morphogenic agent in plants. Auxin accumulation drives a wide range of plant developmental processes including, but

Electronic supplementary material The online version of this article (https://doi.org/10.1140/epjp/ s13360-021-01204-6) contains supplementary material, which is available to authorized users.

a e-mail:k.alim@tum.de (corresponding author)

Published online: 22 February 2021 
not limited to initiation of cell growth, cell division, and cell differentiation [12-14]. Establishment of auxin patterns is ubiquitous in plant organ morphogenesis [15]. The best characterized example is the regular patterns of auxin spots in the outmost epidermal cell layer at the tip of the shoot that prefigures the regular disposition of organs called phyllotactic pattern [16-21]. These auxin accumulation spots mark the location of emerging primordia of new aerial plant organs. Auxin patterns result from the polar distribution of auxin efflux carriers called PIN-FORMED (PINs) [15,16,20,22-24]. Because of its prevalence in plant development, understanding how these auxin patterns emerge has been intensively studied and mathematically modelled. Auxin concentration feedback models [25-29], organize their flow up-the-gradient of auxin concentration, reinforcing auxin maxima. Canalization models, or flux-based models, [30-36] reinforce already existing flows, and, as such, both up-thegradient and down-the-gradient flows can exist. Some attempts at unifying both mechanisms have been made [35,37-39], yet many conditions have to be imposed to explain, for instance, the fountain-like patterns arising during root development $[35,40]$.

Tissue mechanics has emerged as a potent regulator of plant development [5,41-44]. Plant cells are able to read mechanical stress and respond accordingly, rearranging their microtubules along the main direction of mechanical stresses [41]. Furthermore, PIN1 polarity and microtubules alignment at the shoot apical meristem are correlated [10], suggesting the possibility of PIN localisation being mechanically regulated. This hypothetical coupling between PIN localisation and mechanical cues is theoretically able to predict PIN polarity and density for a wide range of cell wall stress and membrane tension [11]. Such coupling is also supported by several other observations: the physical connection of PINs to the cell wall [45], the change in polarity induced by cell curvature [46], and disorganization of PIN polarity by modification of the cell wall mechanical properties [7].

Auxin can induce remodelling of the cell wall and thus modify its mechanical properties $[4,6,7,47]$. This may in turn influence PIN localisation and therefore have consequences on the pattern of auxin. Modelling of this feedback in a tissue showed that mechanical stresses can lead to the emergence of a regular phyllotactic auxin pattern by regulating PIN localisation [10]. Although this result shows the importance of local mechanical coupling (Fig. 1) for emergence of auxin patterns, the full extent of the impact of mechanical coupling on pattern emergence remains unclear.

In fact, cell strain is a compromise between its mechanical properties and the restrictions placed upon its shape by the surrounding cells given the condition that the tissue remains connected. In other words, stiffness variations contribute additional terms to tissue strain. In order to explore the effect of the latter, we adapt the model for auxin transport introduced in [10] to a vertex model mechanical description of the tissue, a tissue-wide mechanical model and compare it to a uncoupled tissue approximation with the same auxin transport model but where we prescribe an average stress acting on all cells (Fig. 2).

By comparing both models, we find that due to stress fields arising from mechanical feedback the magnitude of auxin spots is larger for lower stress-PIN coupling, indicating a more efficient transition between low and high auxin regimes and the subsequent potential cell behaviour response. Furthermore, we explore the information content of auxin distributions when noise is considered and show that tissue-wide mechanical coupling improves robustness of auxin patterns. 


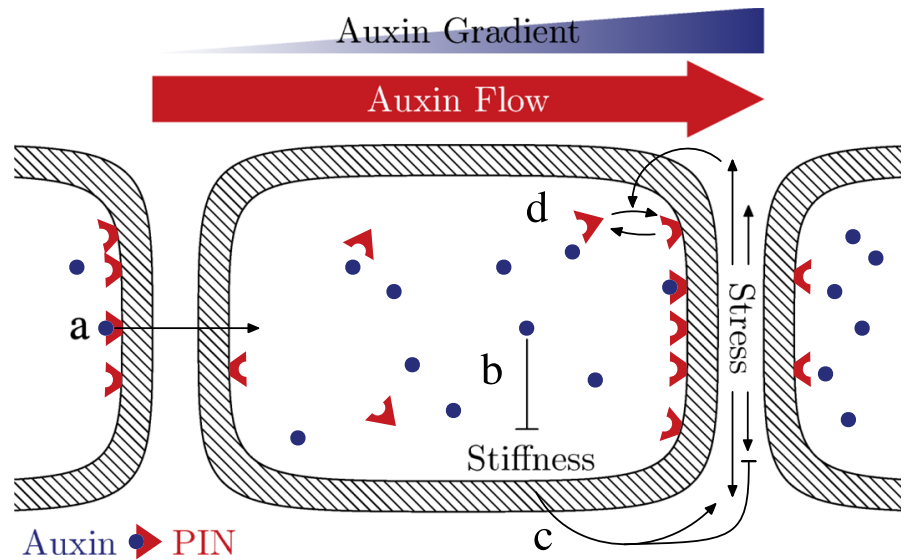

Fig. 1 Schematic representation of the cell-cell feedback mechanism between cell wall loosening via auxin and mechanical control of PIN. (a) Auxin is transported to neighbouring cells via bound PIN efflux carriers. (b) Auxin interacts with the mechanical properties of the cell wall reducing its stiffness. (c) Increasing stiffness of a particular wall component shifts the stress load from the component of its neighbour to itself. (d) Wall stress promotes PIN binding. A difference in auxin, therefore, induces a stress difference between the two compartments separating both cells. This stress difference is such that PIN binds preferentially in the cell with lower auxin concentration, increasing the flow of auxin into the cell with higher auxin concentration
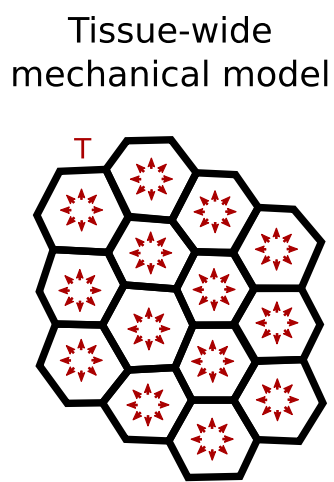

\section{Uncoupled tissue approximation}

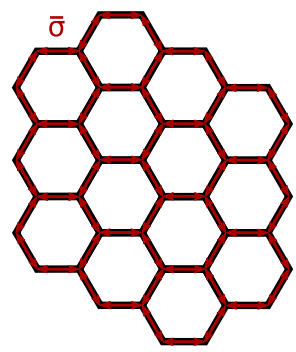

Fig. 2 Schematic difference between the tissue-wide mechanical model (left) and the uncoupled tissue approximation (right). In the tissue-wide mechanical model, turgor pressure, $T$, and stiffness determine the vertex positions that minimize mechanical energy. Wall strain and stress are then inferred from the mechanical configuration. In the approximation, we prescribe average wall stress, $\bar{\sigma}$, with a static geometry. This approximation disregards the effect of stiffness variations on strain. The prescription of stress in the approximation renders the mechanical interaction to be only between nearest neighbours and uncoupled from all other cells. In the tissue-wide mechanical model, the mechanical state is a function of all cells in the tissue

\section{Methods}

In order to investigate the interaction between auxin cell wall softening and collective tissue mechanics, we use a vertex model to describe the mechanical behaviour of the tissue and a compartment model to express auxin concentration and transport between adjacent cells. 

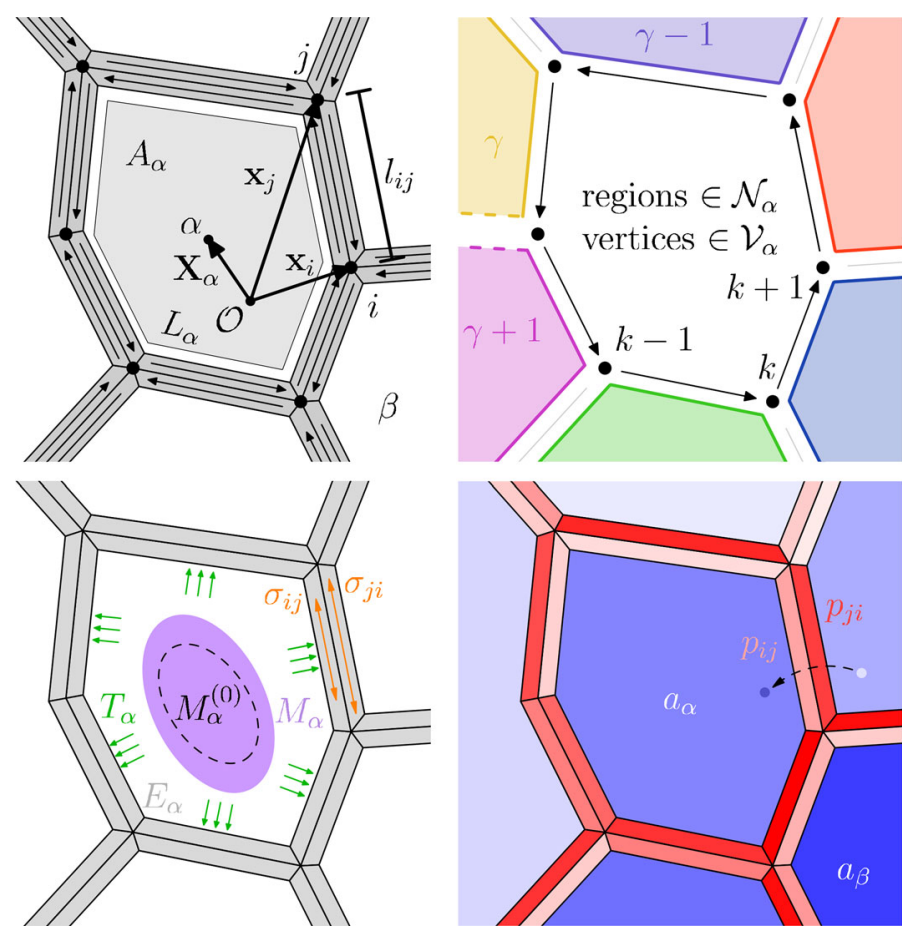

Fig. 3 Vertex model description of a cell as a geometrical, mechanical, and biologically active entity. (top left) A cell $\alpha$ surrounded by its cell walls with centroid $\mathbf{X}_{\alpha}$, area $A_{\alpha}$ and perimeter $L_{\alpha}$. Vertices $i$ and $j$ have positions $\mathbf{x}_{i}$ and $\mathbf{x}_{j}$ and the distance between them is $l_{i j}=l_{j i}$. (top right) Set of surrounding regions, one for each wall, $\mathcal{N}_{\alpha}$, and set surrounding vertices, $\mathcal{V}_{\alpha}$, used in the equations of the model. (bottom left) Mechanically, cell $\alpha$ is under turgor pressure $T_{\alpha}$, the surrounding wall compartments have stiffness $E_{\alpha} \cdot M_{\alpha}$ is the second moment of area of cell $\alpha$, whereas $M_{\alpha}^{(0)}$ is that same quantity when the cell is at rest. $\sigma_{i j}$ refers to the longitudinal stress acting on the compartment of the wall. (bottom right) Cell $\alpha$ has an auxin concentration $a_{\alpha}$ which is expressed, degraded and transported, both passively and actively. The active component of auxin transport relies on the density of membrane-bound efflux auxin carriers facing a particular wall compartment, $p_{i j}$

\subsection{Geometrical set-up of the tissue}

The tissue is described by a tiling of two-dimensional space into $M$ cells surrounded by their cell walls. Walls are represented as edges connecting two vertices each, positioned at $\mathbf{x}_{i}=\left(x_{i}, y_{i}\right), i \in[1, N]$. Here, we reserve Latin indices for vertex numbering and Greek ones for cells. Each cell wall segment has two compartments, one facing each cell. Therefore, we represent each cell wall with two edges of opposite direction, one for each compartment. The position of tissue vertices fully define geometrical quantities such as cell areas, $A_{\alpha}$, cell perimeters, $L_{\alpha}$, wall lengths, $l_{i j}=l_{j i}$, and cell centroids, $\mathbf{X}_{\alpha}$ (Fig. 3 top left). To simplify notation significantly, we also define for each cell the cyclically ordered set of all vertices around that cell, $\mathcal{V}_{\alpha}$, arranged counterclockwise (ccw). Hence, we use $\sum_{i \in \mathcal{V}_{\alpha}}$ to signify the sum over all vertices surrounding cell $\alpha$ with an arbitrary start, where $i+1$ and $i-1$ mean, respectively, the next and previous ccw vertex. Similarly, we introduce $\mathcal{N}_{\alpha}$ as the cyclically ordered (counterclockwise) set of all neighbouring regions around cell $\alpha$, one for each edge of $\alpha$ (Fig. 3 top right). 


\subsection{Tissue mechanics-tissue-wide coupling}

Vertex models are a widely employed theoretical approach to describe mechanics of epithelial tissues and morphogenesis [9,48-53]. The essence of vertex models is that cell geometry within a tissue is given as the mechanical equilibrium of the tissue. In the case of plant cells, the shape of a cell is a competition between the turgor pressure, $T_{\alpha}$, all cells exert on each other and the cell's resistance to deformation with stiffness, $E_{\alpha}$. Strain acting on each cell will be described using the second moment of area of the corresponding cell in reference to its centroid, $M_{\alpha}$, whose components are

$$
\begin{aligned}
& M_{\alpha_{x x}}=\sum_{i \in \mathcal{V}_{\alpha}} \frac{n_{i}}{12}\left(x_{i}^{\prime^{2}}+x_{i}^{\prime} x_{i+1}^{\prime}+x_{i+1}^{\prime 2}\right), \\
& M_{\alpha_{y y}}=\sum_{i \in \mathcal{V}_{\alpha}} \frac{n_{i}}{12}\left(y_{i}^{\prime 2}+y_{i}^{\prime} y_{i+1}^{\prime}+y_{i+1}^{\prime 2}\right), \\
& M_{\alpha_{x y}}=M_{\alpha_{y x}}=\sum_{i \in \mathcal{V}_{\alpha}} \frac{n_{i}}{24}\left(x_{i}^{\prime} y_{i+1}^{\prime}+2 x_{i}^{\prime} y_{i}^{\prime}+2 x_{i+1}^{\prime} y_{i+1}^{\prime}+x_{i+1}^{\prime} y_{i}^{\prime}\right),
\end{aligned}
$$

where the primed coordinates represent the translation transformation, $\mathbf{x}_{i}^{\prime}=\left(x_{i}^{\prime}, y_{i}^{\prime}\right)=$ $\mathbf{x}_{i}-\mathbf{X}_{\alpha}$, and $n_{i}=x_{i}^{\prime} y_{i+1}^{\prime}-x_{i+1}^{\prime} y_{i}^{\prime}, i \in \mathcal{V}_{\alpha}$. Given a rest shape matrix, $M_{\alpha}^{(0)}$, we define cell strain as the normalized difference between both matrices,

$$
\varepsilon_{\alpha}=\frac{M_{\alpha}-M_{\alpha}^{(0)}}{\operatorname{Tr}\left(M_{\alpha}^{(0)}\right)},
$$

and stress with $\sigma_{\alpha}=E_{\alpha} \varepsilon_{\alpha}$. Having described the tissue mechanically (Fig. 3 bottom left), we define the energy for a single cell as the sum of work done by turgor pressure and elastic deformation energy, resulting in the tissue mechanical energy,

$$
\mathcal{H}=\sum_{\alpha=1}^{M}\left[\frac{1}{2} A_{\alpha} E_{\alpha} \frac{\left\|M_{\alpha}-M_{\alpha}^{(0)}\right\|_{2}^{2}}{\operatorname{Tr}^{2}\left(M_{\alpha}^{(0)}\right)}-A_{\alpha} T_{\alpha}\right] .
$$

Using this model, we obtain the shape of the tissue by minimizing $\mathcal{H}$ with respect to vertex positions.

After minimizing (Eq. 5), we quantify the stress acting on each wall through the average strain acting on each cell given by (Eq. 4). Assuming that cell wall rest length is the same between two adjacent wall compartments then it follows that they are under the same longitudinal strain, which is, to first approximation, the average between the two cells surrounding them. Therefore, longitudinal average strain acting on a specific wall used here is

$$
\bar{\varepsilon}_{\alpha \beta}=\bar{\varepsilon}_{\beta \alpha} \sim \hat{\mathbf{t}}_{\alpha \beta}^{T} \frac{\varepsilon_{\alpha}+\varepsilon_{\beta}}{2} \hat{\mathbf{t}}_{\alpha \beta},
$$

where $\mathbf{t}_{\alpha \beta}$ is a unit vector along the wall separating cell $\alpha$ and cell $\beta$. Note that this interpolation assumes a continuous strain field. Then the stresses acting on each compartment are by the constitutive equation of a linear elastic isotropic material with Poisson ratio $v=0$,

$$
\sigma_{\alpha \beta}=E_{\alpha} \bar{\varepsilon}_{\alpha \beta} \neq \sigma_{\beta \alpha}=E_{\beta} \bar{\varepsilon}_{\beta \alpha} .
$$

Note that we are only considering the longitudinal components with regards to the cell wall, which means that $\bar{\varepsilon}_{\alpha \beta}$ and $\sigma_{\alpha \beta}$ are scalar quantities. More details on the mechanical model 
used can be found in the supporting text. As argued in the supporting material, our choice of $v=0$ does not impact the qualitative behaviour studied here.

\subsection{Tissue mechanics-uncoupled tissue approximation}

To assess the impact of collective mechanical behaviour within a tissue on auxin pattern selforganization, we approximate the tissue-wide mechanical model to a static tissue geometry where we approximate the effects of turgor pressure of each individual cells in the static tissue by a constant average stress $\bar{\sigma}$ acting on it [10]. Again assuming that both wall compartments have the same rest length, we infer that the stress acting on a particular wall depends only on $\bar{\sigma}$ and the stiffness of the adjacent cells. Effectively, the average longitudinal strain acting on a wall surrounded by cells $\alpha$ and $\beta$ would simply be

$$
\bar{\varepsilon}_{\alpha \beta}=\bar{\varepsilon}_{\beta \alpha}=2 \bar{\sigma} /\left(E_{\alpha}+E_{\beta}\right) .
$$

This way, instead of minimizing the full mechanical model (Eq. 5) given a set of turgor pressures $T_{\alpha}$ and rest shape matrices $M_{\alpha}^{(0)}$ we can, in the static tissue, immediately compute stress with Eq. 7 yielding,

$$
\sigma_{\alpha \beta}=\frac{2 E_{\alpha} \bar{\sigma}}{E_{\alpha}+E_{\beta}}
$$

Interestingly, Eq. 9 is valid for $v \neq 0$ as demonstrated in the supporting material.

In order to compare the two models, we choose the value of $\bar{\sigma}$ to be the same as the stress obtained through minimisation of (Eq. 5), for a given set of $T_{\alpha}$ and $M_{\alpha}^{(0)}$, with the constraint of the same end geometry.

Note that not only can this approximation be interpreted as the tissue being mechanically coupled only to the nearest neighbours, disregarding the rest of the tissue, (Fig. 2), but also as an analogous non-mechanical auxin concentration feedback model.

\subsection{Auxin transport-compartment model}

Compartment models for auxin transport are well adapted to the context of plant development, since the prerequisite of a boundary of a plant cell is particularly well defined by courtesy of the cell wall.

Although passive diffusion occurs across cell walls, the dominant players in auxin transport are membrane-bound carriers [22,24]. Namely, efflux transporters of the PIN family are important due to their anisotropic positioning around a cell [16], which leads to a net auxin flow from one cell to the next. Let $a_{\alpha}$ denote an non-dimensional and normalized average auxin concentration inside cell $\alpha$. Following the model by [10], which is similar to previous mathematical models [25,26,29], auxin evolves according to auxin metabolism in the cell, passive diffusion between cells and active transport across cell walls via PIN,

$$
\begin{aligned}
\frac{\mathrm{d} a_{\alpha}}{\mathrm{d} t}= & \gamma^{*}-\delta^{*} a_{\alpha}+\mathcal{D} \sum_{\beta \in \mathcal{N}_{\alpha}} W_{\alpha \beta}\left(a_{\beta}-a_{\alpha}\right) \\
& +\mathcal{P} \sum_{\beta \in \mathcal{N}_{\alpha}} W_{\alpha \beta}\left(p_{\beta \alpha} \frac{a_{\beta}}{K+a_{\beta}}-p_{\alpha \beta} \frac{a_{\alpha}}{K+a_{\alpha}}\right),
\end{aligned}
$$

where $\gamma^{*}$ is the auxin production rate, $\delta^{*}$ is the auxin decay rate, $W_{\alpha \beta}=l_{\alpha \beta} / A_{\alpha}$, with $K$, $\mathcal{P}$, and $\mathcal{D}$ as adjustable parameters. $\mathcal{D}$ is the passive permeability of plant cells, whereas $\mathcal{P}$ is permeability of the cell wall due to PIN-mediated transport of auxin, and $K$ is the Michaelis- 
Menten constant for the efflux of auxin. More information on how this expression is derived can be found in the supporting text. Although this description ignores the auxin present within the extracellular domain and inside the cell wall, it has been shown that under physiological assumptions, this is a valid approximation [29]. The active transport term depends on the amount of bound PIN in each cell wall,

$$
p_{\alpha \beta}=\frac{f_{\alpha \beta}}{1+\sum_{\gamma \in \mathcal{N}_{\alpha}} \frac{l_{\alpha \gamma}}{L_{\alpha}} f_{\alpha \gamma}}, \beta \in \mathcal{N}_{\alpha},
$$

where $f_{\alpha \beta}, \beta \in \mathcal{N}_{\alpha}$ expresses the ratio between binding and unbinding rates of a particular wall (Fig. 3 bottom right). Note that $p_{\alpha \beta}$ is different from wall to wall and from cell to cell. This means that in general, $p_{\alpha \beta} \neq p_{\beta \alpha}$, or equivalently, $p_{i j} \neq p_{j i}$. This is consistent with the fact that there are two compartments to a cell wall shared by two adjacent cells. Expression (Eq. 11) is based on the assumption that cell walls around a particular cell compete for the same pool of PIN molecules and that the amount of PIN scales with cell perimeter. This competition has been shown to be important in the polarization of PIN [29]. Alternatively, one could also scale the amount of PIN with cell size or not scale it at all. In the former case, smaller cells would be slightly preferred for auxin accumulation, whereas in the latter, larger cells would be preferred instead. Since we want to study the impact of stress patterns on the tissue, we want to decouple it from this effect as much as possible, choosing instead to scale the amount of PIN with perimeter.

The trivial fixed point of these dynamical equations is given by $a_{\alpha}=\mu^{*} / \delta^{*}, \forall \alpha$, which also results in equal PIN density across all walls, provided turgor pressure $T_{\alpha}$ and stiffness $E_{\alpha}$ are the same across the tissue.

The feedback between tissue mechanics and auxin pattern unfolds as auxin transport affects tissue mechanics due to auxin, $a_{\alpha}$, controlling cell wall stiffness, $E_{\alpha}$, and in reverse tissue stress, $\sigma_{\alpha}$, affects auxin transport by regulating PIN binding rates, $f_{\alpha \beta}$, as hypothesized by $[10,11]$.

\subsection{Mechanical regulation of PIN binding}

According to the hypothesis presented by $[10,11]$, mechanical cues up-regulate PIN binding. The distinction between whether these mechanical cues are strain or stress has been studied recently by [54], yet the exact nature remains unclear. Following the model presented by [10], we model the binding-unbinding ratio, $f_{\alpha \beta}$, as being a power law on positive stress,

$$
f_{\alpha \beta}=f\left(\sigma_{\alpha \beta}\right)= \begin{cases}\eta\left(\sigma_{\alpha \beta}\right)^{n}, & \sigma_{\alpha \beta}>0, \\ 0, & \sigma_{\alpha \beta} \leq 0,\end{cases}
$$

where the stresses, $\sigma_{\alpha \beta}$, follow from tissue mechanics after minimization of the full mechanical model (Eq. 5), or, in the averaged stress approximation, it is the stress load on that particular compartment given by (Eq. 9). Furthermore, $n$ is the exponent of this power law, and $\eta$ captures the coupling between stress and PIN. Effectively, this mechanical coupling to PIN parameter corresponds to the sensing and subsequent response to stress, loosely translating into how much resources the cell needs to spend for processing stress cues.

\subsection{Auxin-mediated cell wall softening}

Auxin affects the mechanical properties of a cell wall via methyl esterification of pectin $[6,7]$, resulting in a decrease of the stiffness of the cell wall. We assume that all cell wall 


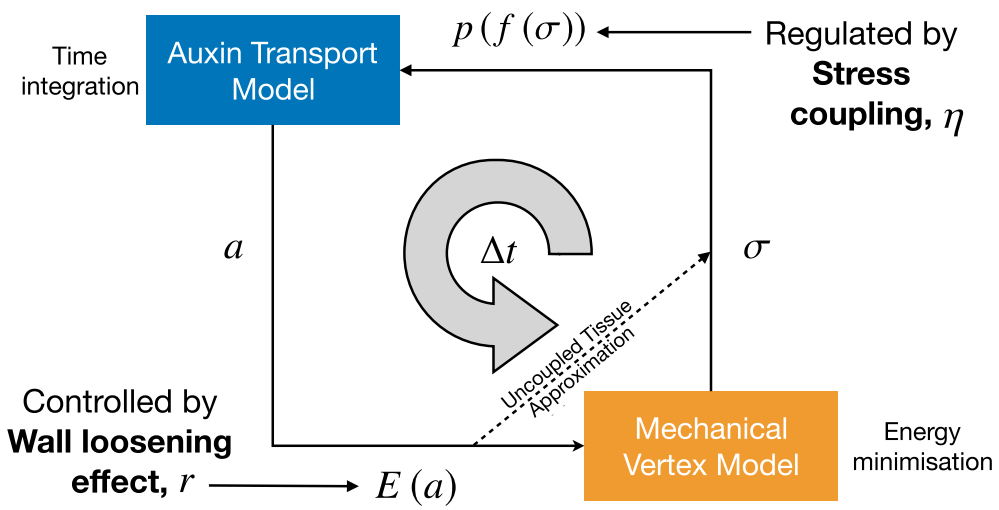

Fig. 4 Schematic representation of the time evolution of the model. From mechanical relaxation of the mechanical model, we calculate PIN densities on each wall via stress. Then we integrate auxin dynamics for a time step and update the stiffness of each cell. This process knocks the system out of the previous mechanical energy minimum, and it has to be relaxed again. Alternatively, we can shortcut energy minimization using the averaged stress approximation for a static tissue. This procedure is repeated until $t=t_{\max }$. The parameters $r$, wall loosening effect, and $\eta$, stress coupling, interface both models and are, therefore, of critical importance to the mechanism studied

compartments surrounding cell $\alpha$ share the same stiffness, $E_{\alpha}$. To capture this effect, we model stiffness with a Hill function [10],

$$
E_{\alpha}=E\left(a_{\alpha}\right)=E_{0}\left(1+r \frac{1-a_{\alpha}^{m}}{1+a_{\alpha}^{m}}\right),
$$

where $r \in[0,1$ [ which we define as the cell wall loosening effect, $m$ is the Hill exponent of this interaction, and $E_{0}$ is the stiffness of the cell walls when its auxin concentration is $a_{\alpha}=1$. At low values of auxin, $E_{\alpha}$ approaches the value $(1+r) E_{0}$, whereas at high auxin concentration, $E_{\alpha}$ approaches $(1-r) E_{0}$. Given a distribution of auxin, we can compute the wall stiffness in (Eq. 5) from (Eq. 13), or the stress acting on a specific compartment in (Eq. 9) for the approximated model.

\subsection{Integrating auxin transport and tissue mechanics}

At each time step, $\Delta t$, starting from an auxin distribution, we compute the stiffness of each cell according to (Eq. 13). Then, with the input of all turgor pressures, we minimize (Eq. 5) to obtain tissue geometry and stresses acting on each wall. Auxin concentration in each cell will evolve according to (Eq. 10), where the active transport term will be regulated by stress according to (Eq. 12) via (Eq. 11). A new auxin distribution will result at the end of this iteration, and we will be ready to take another time step (Fig. 4). We repeat this process until $t=t_{\max }$.

\subsection{Implementation}

We implemented this model with $\mathrm{C}++$ programming language, where we have used the Quad-Edge data structure for geometry and topology of the tissue [55], implemented in the library Quad-Edge [56]. In order to minimize the mechanical energy of the tissue, we have used a limited-memory Broyden-Fletcher-Goldfarb-Shanno algorithm (L-BFGS) [57, 58], implemented in the library NLopt [59]. For solving the set of ODEs presented in the 
compartment model, we used the explicit embedded Runge-Kutta-Fehlberg method (often referred to as RKF45) implemented in the GNU Scientific Library (GSL) [60]. We wrapped the resulting classes into a python module with SWIG. For additional details regarding the parameters used for the simulations of the following section, consult Table S1 in the supporting material.

\subsection{Observables}

In order to quantify the existence of auxin patterns, we compute the difference between an emerging auxin concentration pattern and the trivial steady state of uniform auxin concentration pattern defined as $a_{\alpha}=\gamma^{*} / \delta^{*}, \forall \alpha$. To account for a large range of orders of magnitude of auxin concentration, we consider as an order parameter,

$$
\varphi=\frac{\left\langle\ln ^{2}\left(a_{\alpha}\right)\right\rangle_{M}}{\delta^{2}+\left\langle\ln ^{2}\left(a_{\alpha}\right)\right\rangle_{M}},
$$

where $\langle\cdot\rangle_{M}$ denotes an average over all cells within the tissue. This way, $\varphi \approx 0$ means that there are no discernible patterns, whereas $\varphi \approx 1$ implies prominent auxin patterning. The term $\delta^{2}$ defines the sensitivity of this measure, such that an average deviation of $\delta$ yields $\varphi \approx 1 / 2$ (for small $\delta$ ). We will choose $\delta=0.1$, i. e. , a $10 \%$ deviation from the trivial steady state.

We also keep track of the average of auxin above basal levels in order to gauge the potential degree of modulation of auxin-mediated cell behaviour.

Furthermore, to characterize cells with regards to PIN localization we introduce the magnitude of the average PIN efflux direction,

$$
F_{\alpha}=\left\|\sum_{i \in \mathcal{V}_{\alpha}} \frac{l_{i i+1}}{L_{\alpha}} p_{i i+1} \hat{\mathbf{n}}_{i i+1}\right\|,
$$

where $\hat{\mathbf{n}}_{i+1}$ is the unit vector normal to the wall pointing outwards from $\alpha$.

Aside from a global measure of auxin patterning, it is also important to locally relate auxin to tissue mechanics. Namely, for auxin we are interested in auxin concentration, $a_{\alpha}$, and auxin local gradient, obtained by interpolation,

$$
\nabla a_{\alpha}=\frac{1}{2 A_{\alpha}^{*}} \sum_{\gamma \in \mathcal{N}_{\alpha}}\left(\begin{array}{cc}
Y_{\gamma+1}^{\prime} & -Y_{\gamma}^{\prime} \\
-X_{\gamma+1}^{\prime} & X_{\gamma}^{\prime}
\end{array}\right)\left(\begin{array}{c}
a_{\gamma}-a_{\alpha} \\
a_{\gamma+1}-a_{\alpha}
\end{array}\right)
$$

where $\mathbf{X}_{\gamma}^{\prime}=\left(X_{\gamma}^{\prime}, Y_{\gamma}^{\prime}\right)=\mathbf{X}_{\gamma}-\mathbf{X}_{\alpha}$ and

$$
A_{\alpha}^{*}=\frac{1}{2} \sum_{\gamma \in \mathcal{N}_{\alpha}}\left(X_{\gamma}^{\prime} Y_{\gamma+1}^{\prime}-Y_{\gamma}^{\prime} X_{\gamma+1}^{\prime}\right) .
$$

In fact, the quantity $\left|\nabla a_{\alpha}\right|$ can be used as an indicator of whether there is an interface between auxin spots and the rest of the tissue.

With regards to tissue mechanics, the local quantities we quantify are the isotropic component of stress,

$$
P_{\alpha}=\frac{1}{2} \operatorname{Tr}\left(\sigma_{\alpha}\right)
$$


and the stress deviator tensor projected along the direction of the auxin gradient,

$$
D_{\alpha}=\frac{\nabla a_{\alpha}^{T} \sigma_{\alpha}^{\prime} \nabla a_{\alpha}}{\left|\nabla a_{\alpha}\right|^{2}}
$$

where $\sigma_{\alpha}^{\prime}=\sigma_{\alpha}-I P_{\alpha}$, and $I$ is the identity matrix. Therefore, $P_{\alpha}$ is a measure if a cell is being compressed $\left(P_{\alpha}<0\right)$, or pulled apart $\left(P_{\alpha}>0\right)$, and $D_{\alpha}$ translates into if a cell is more compressed along the auxin gradient than perpendicular to it $\left(D_{\alpha}<0\right)$, or vice-versa $\left(D_{\alpha}>0\right)$.

Finally, to measure the disruption of an auxin pattern we approximate entropy by means of a Riemann sum,

$$
S[\Pi]=-\sum_{i=-\infty}^{\infty} \Pi(i \Delta a) \Delta a \ln (\Pi(i \Delta a) \Delta a),
$$

where $\Pi(a)$ is the probability density function of auxin and $\Delta a$ the partition size. Note that it is only meaningful to compare entropy measures obtained with the same partition size $\Delta a$. Here, the probability density function of auxin concentration is obtained by applying a kernel density estimation on the resulting tissue auxin values. Note that $\Pi(a)$ is a continuous function. In order to infer it from simulation data, for each auxin value in the tissue, $a_{\alpha}$, we add Kernel functions $K_{w}(a)$, obeying $\int_{-\infty}^{\infty} K_{w}(a) d a=1$ and $K_{w}(a)=K_{w}(-a)$. Then we can estimate

$$
\Pi(a) \sim \frac{1}{M} \sum_{\alpha=1}^{M} K_{w}\left(a-a_{\alpha}\right),
$$

where $w$ is a smoothing parameter defining the width of the Kernel, this parameter is sometimes called bandwidth. This statistical tool is called kernel density estimation (KDE) [61]. We use the Epanechnikov kernel because it is bounded and we can force $\Pi(a)=0, a \leq 0$.

\section{Results}

3.1 The tissue-wide mechanical model captures stress patterns after ablation

First we verify that the tissue-wide mechanical model captures the expected mechanical behaviour and auxin patterning when a cell is ablated. To model ablation, we set the stiffness of the ablated cell walls to $E_{0}=0$, block all auxin transport to and from it, block PIN transporters of adjacent cells from binding to the shared wall with the ablated cell, and, finally, we lower the turgor pressure to only $10 \%$ of the original value. This remnant of pressure represents the surface tension emerging from pressure of the inner layers of the shoot apical meristem acting on a curved surface, as required by the Young-Laplace equation. This is necessary since the model only simulates the epidermal layer in a plane.

We observe that the region neighbouring the ablation site gets depleted of auxin due to PIN binding preferentially to the walls circumferentially aligned around the ablated cell in accordance with the stress principal directions (Fig. 5a). This stress pattern is in agreement with calculations performed by [41] in this setting and PIN aligns according to the ablation experiments in [10].

We also simulated different wound shapes. The resulting stress patterns are shown the supporting material. Stress directions align along the shape of the ablation wound. 

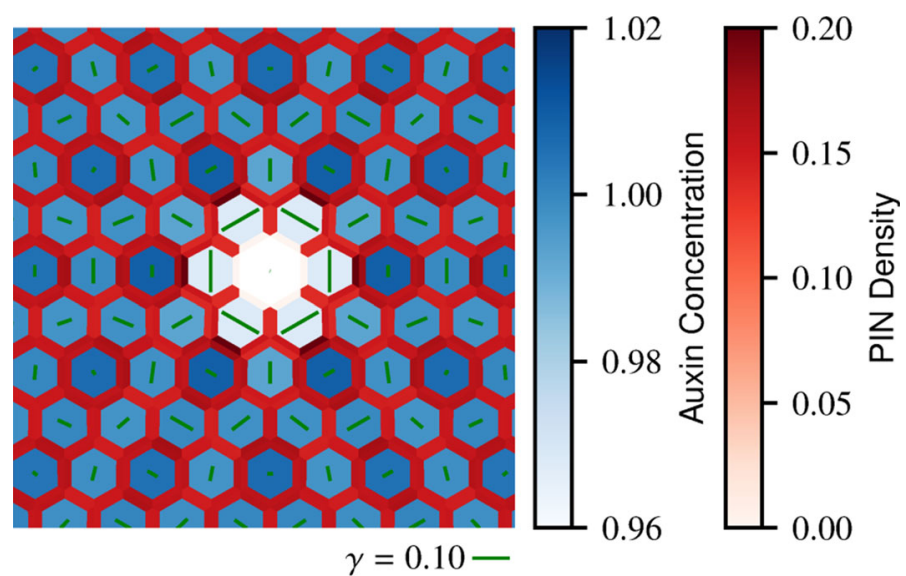

Fig. 5 Tissue-wide mechanical model captures expected stress patterns as well as auxin and PIN distribution after ablation. Green lines represent the magnitude and direction of principal stress, measured $\gamma=\frac{\lambda_{+}-\lambda_{-}}{\lambda_{+}+\lambda_{-}}$, where $\lambda_{ \pm}$are the largest and lowest eigenvalues of the stress tensor. The ablation perturbs auxin patterning by redirecting PIN. This PIN reorientation coincides with the circumferential stress patterns around the ablation site, as seen in experiments and simulations [10,41]. $r=0.65$ and $\eta=1.5$

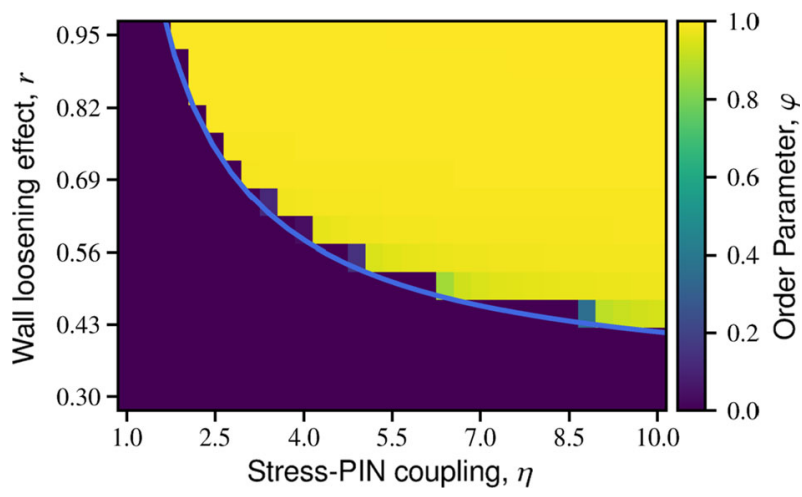

Fig. 6 Simulation results of the order parameter $\varphi$, indicator for the existence of auxin patterns, as a function of $r \in[0.30,0.95]$ and $\eta \in[1.0,10.0]$ for a model with tissue-wide stress patterning. The simulated tissue is composed of 2977 initially hexagonal cells. The blue line represents the analytically predicted instability for the uncoupled tissue approximation (Eq. 22)

Thus our mechanical model faithfully capture the typical tissue behaviour upon ablation with regards to stress, auxin and PIN transporter patterns.

\subsection{Conditions for auxin patterns emergence}

The uncoupled tissue approximation allows to analytically compute the conditions for spontaneous auxin pattern emergence in a general regular lattice (Fig. 6). Effectively, for a regular grid, the condition for pattern formation is,

$$
\mathcal{M}>\frac{K+1}{W \mathcal{P}}\left[1+\sqrt{1+2 W\left(\frac{\mathcal{P} K}{(K+1)^{2}}+\frac{\mathcal{D}}{p_{0}}\right)}\right]^{2},
$$


where $\mathcal{M}=n m r, W=4 / \sqrt{3}$ is a geometrical factor specific to the used grid, and $p_{0}=$ $f(\bar{\sigma}) /(1+f(\bar{\sigma}))$ (see supporting material for the linear stability analysis details). Equation 22 is the closed form of more general expressions presented by [10,29] tailored to our system and parameters.

To quantify the existence of auxin patterns in the model with tissue-wide stress patterning, we computed the order parameter $\varphi$ defined in Eq. 14 for simulations with different values of wall loosening effect $r$ and stress coupling $\eta$ (Fig. 6). These two parameters are conceptually important since the former is the cause for stiffness inhomogeneity of the tissue, and the latter represents a plant cell's sensitivity to mechanical cues.

We observe a very good agreement between the conditions for pattern emergence (Eq. 22) analytically predicted in the case of the uncoupled tissue approximation and the transition of $\varphi$ in the case of tissue-wide stress patterning (Fig. 6). This means that at the onset of patterns emergence the auxin concentrations are similar enough to make the assumption that the effect of turgor pressure is simply an isotropic stress across the entire tissue, validating the approximation near the transition. This observation is in agreement with the auxin pattern emergence mechanism hypothesis by [10] (Fig. 1). The agreement between the two models does not necessarily apply after patterns emerge. This poses the question of the role of mechanics in potentially enhancing or hindering auxin flows.

\subsection{Global mechanical response reinforces PIN polarity}

To understand the role of tissue-wide stress patterning on the emergence of PIN-driven auxin patterns, we quantify how PIN rearranges in the model with tissue-wide stress patterning versus the uncoupled tissue approximation.

We compute the average PIN efflux direction, i.e., average PIN polarity for each combination of the parameters $r$ (auxin-induced cell wall loosening) and $\eta$ (coupling of PIN to stress) under the approximated (Fig. 7 top left) and tissue-wide (Fig. 7 top right) stress coupling regimes.

We observe an overall increase in PIN polarity in the tissue-wide stress coupling regime compared with the uncoupled tissue approximation. PIN polarity also becomes more sensitive to $r$. For very low values of $r$, tissue stress patterns are slightly detrimental to auxin patterning. These data show that saturation of PIN polarity happens earlier with respect to $\eta$ for intermediate values of $r$. For high values of $r$, we observe a non-monotonic dependence of polarity on $\eta$, effectively translating into an optimal value of $\eta$.

Visual inspection of the simulations results reveals higher PIN density in proximity of auxin spots and an increase in magnitude of these auxin peaks upon tissue-wide stress patterning (Fig. 7a-d). Moreover, we observe a severe alteration of pattern size and wavelength between both models (Fig. 7 bottom left).

These results show that tissue-wide stress patterning reinforces PIN polarity and that auxin spots are sharper. Next we will quantify how much sharper these auxin spots become.

\subsection{Tissue-wide coupling induces efficient emergence of auxin spots}

Auxin levels in the shoot apical meristem have been shown to affect cell fate reliably [20], even if the flexibility of the auxin signalling mechanism allows for many potential outcomes [62]. We explore auxin spot concentration achieved by both models in order to gauge the impact of tissue-wide stress patterns on the distinguishability of primordium cells.

For this, we first characterize quantitatively the auxin spot average concentration measured for each simulation of the uncoupled tissue approximation (Fig. $8 \mathrm{left}$ ) and tissue-wide 

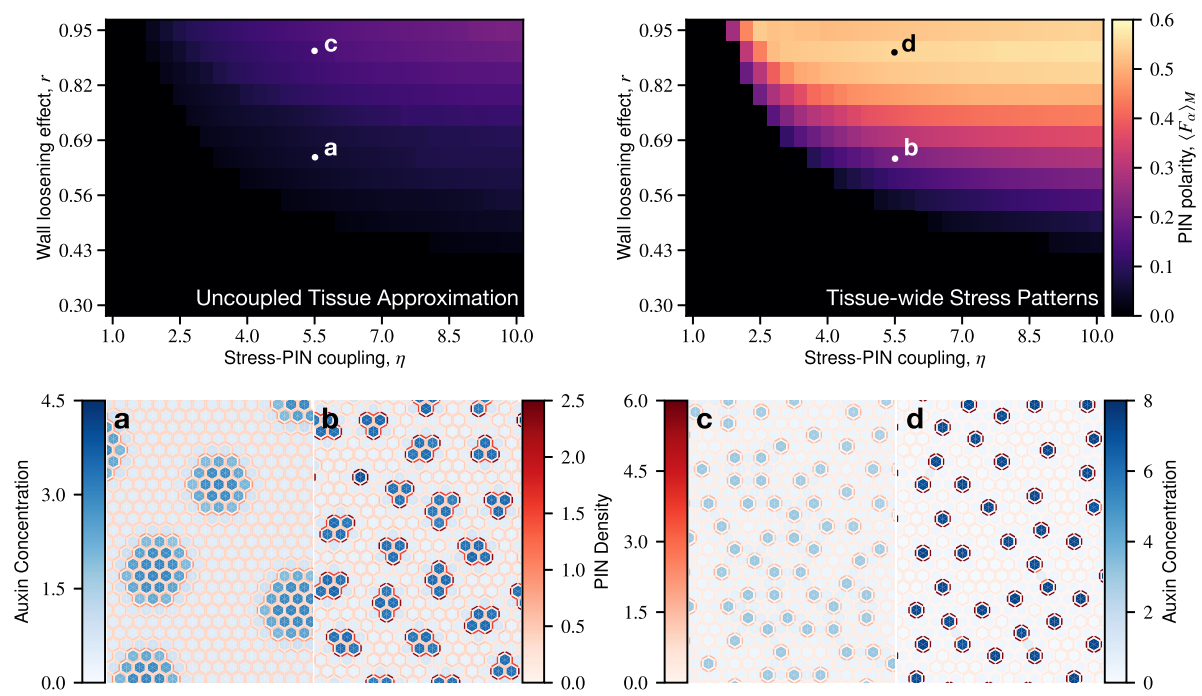

Fig. 7 Quantification of PIN polarity in both models reveals more focused auxin spots due to tissue-wide integration via mechanical coupling. (top) Average magnitude of PIN polarity, $\left\langle F_{\alpha}\right\rangle_{M}$, as a function of stressPIN coupling, $\eta$, and wall loosening effect, $r$, for (top left) the uncoupled tissue approximation and (top right) for the tissue-wide stress patterning. PIN polarity magnitude increases when considering the mechanics of the whole tissue, with a particularly strong dependence on the wall loosening affect $r$ of auxin. The labels represent the parameters plotted for $(\mathrm{a}, \mathrm{b}, \mathrm{c}, \mathrm{d})$ comparison between example results of auxin concentration and PIN density of simulations using the uncoupled tissue approximation ( $a, c)$ and the tissue-wide stress patterning (b, d), for the same value of $\eta=5.5$, and $r=0.65$ (bottom left) or $r=0.90$ (bottom right). In both instances, we observe that PIN polarity and auxin concentration are higher upon tissue-wide stress patterning $(\mathrm{b}, \mathrm{d})$
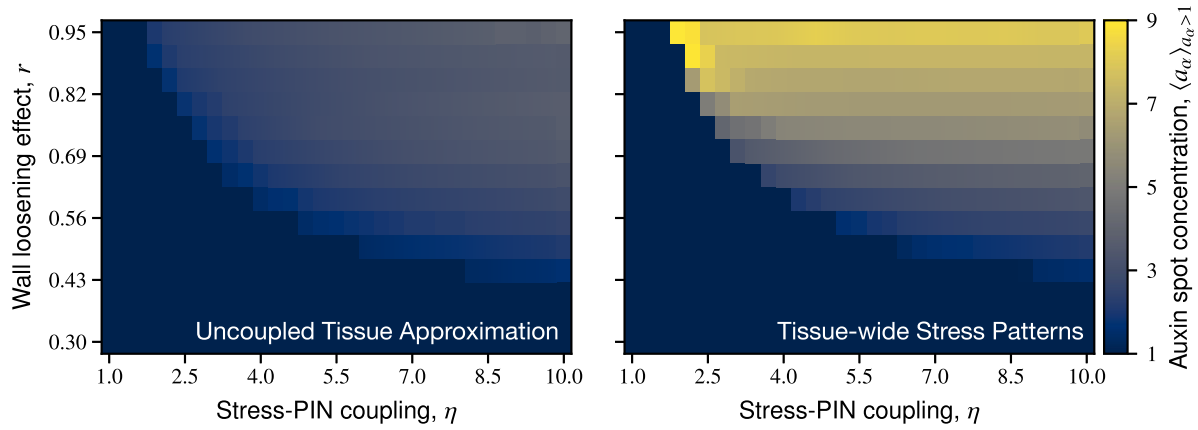

Fig. 8 Characterization of auxin spot concentration reveals more focused auxin spots due to tissue-wide integration via mechanical coupling. Average auxin concentration for cells above basal auxin concentration $\left(a_{\alpha}>1\right)$, for the uncoupled tissue approximation (left), and upon tissue-wide stress patterning (right), as a function of stress-PIN coupling, $\eta$, and wall loosening effect, $r$. Spot auxin concentration increases with both $\eta$ and $r$ in (left); however, in (right), it increases predominantly with $r$. For medium to high values of $r$, auxin concentration jumps to several times immediately after emergence

stress coupling (Fig. 8 right) regimes. We use, as a proxy, the average of cells with auxin concentration $a_{\alpha}>1$ to identify auxin spots.

We observe that the dependence on the parameter $r$ recognized for PIN polarity translates into auxin spot concentration. For medium to high values of $r$, auxin concentration is several 

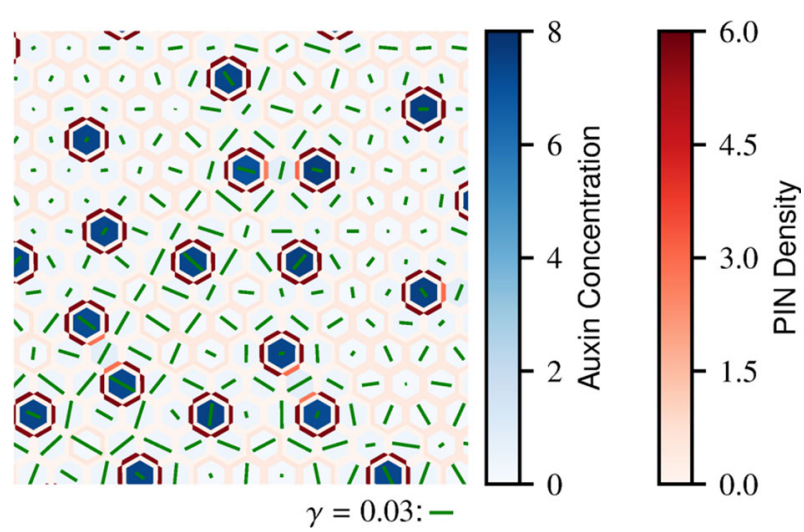

Fig. 9 Map of auxin distribution and PIN density aligns with stress direction. Green lines represent principal direction of stress, measured as $\gamma=\frac{\lambda_{+}-\lambda_{-}}{\lambda_{+}+\lambda_{-}}$, where $\lambda_{ \pm}$are the largest and lowest eigenvalues of the stress tensor. We observe that stress directions in part congruent with auxin spots. $r=0.90$ and $\eta=5.5$

times higher when accounting for tissue-wide behaviour than when considering the uncoupled tissue approximation.

Additionally, at the onset of pattern formation for medium to high values of $r$, we observe a considerable jump in average auxin spot concentration for a small change in $\eta$. This increase in sensitivity to a change in $\eta$ of the system, under the aforementioned conditions, implies a boost in mechanosensing capabilities when considering tissue-wide stress patterning.

Our results point to stress patterns being responsible for the enhancement of auxin spot concentration and flows. In order to make sure we understand why, we decided to observe and quantify stress patterns and their connection to auxin distribution.

\subsection{Part of wall stress within spots is borne by walls at the interface}

In order to analyse tissue-wide stress patterns, we choose an example that has simple auxin patterns that allow for a straightforward interpretation. Under this condition, we choose the parameters $r=0.90$ and $\eta=5.5$ already presented in Fig. 7d, for which we plot on it a measure of anisotropy along the largest principal stress direction (Fig. 9). Here it becomes apparent that stress patterns are related, even if not absolutely, to auxin spot patterns.

To explore this further, we quantify several local quantities, such as auxin concentration, $a_{\alpha}$, auxin gradient norm (Eq. 16), $\left|\nabla a_{\alpha}\right|$, isotropic stress component (Eq. 18), $P_{\alpha}$, and deviator stress tensor projection onto auxin gradient (Eq. 19), $D_{\alpha}$. For the example mentioned above, we record the histograms of the simultaneous occurrence of the pairs $\left(a_{\alpha}, P_{\alpha}\right)$ (Fig. 10 top left) and $\left(\left|\nabla a_{\alpha}\right|, D_{\alpha}\right)$ (Fig. 10 top right).

We can section the results according to high or low auxin concentration (Fig. 10 bottom left), and high or low auxin gradient (Fig. 10 bottom right). Here, high auxin cells are a proxy for auxin spot cells, and high auxin gradient cells are a proxy for cells neighbouring auxin spots. Taking into account that in the uncoupled tissue approximation $P_{\alpha}=\bar{\sigma}$ and $D_{\alpha}=0$ by construction, we can get a better picture of tissue-wide stress patterns.

We observe from data (Fig. 10 bottom left) that $P_{\alpha}$ in cells of auxin spots is lower than in the uncoupled tissue approximation and accompanied by a slight shift in the opposite direction of the $P_{\alpha}$ of the remaining cells. Additionally, we register a noticeable shift towards negative $D_{\alpha}$ for high auxin gradient cells (Fig. 10 bottom right). 

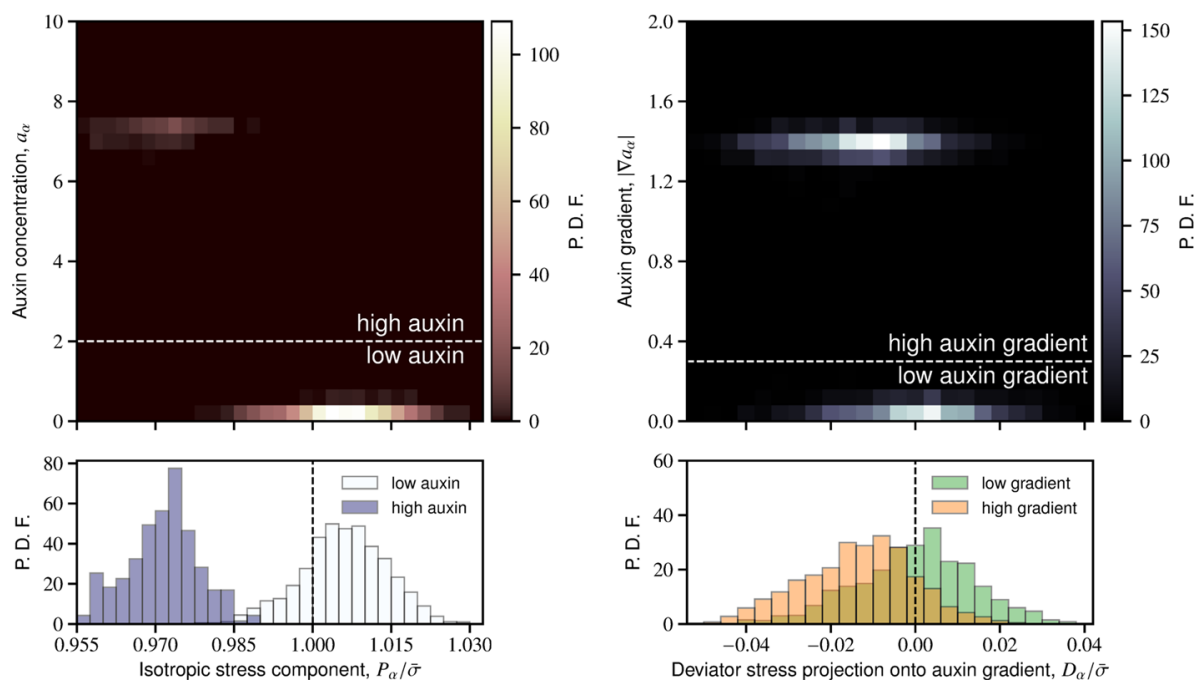

Fig. 10 Stress pattern self-organization concomitant with auxin patterns. Probability density functions (P.D.F.s) of (top left) auxin concentration and isotropic stress component and (top right) auxin gradient magnitude and deviator stress tensor projection onto auxin gradient. In each case, we can identify two populations of cells: high and low auxin concentration (bottom left), and high and low auxin gradient (bottom right). (bottom left), since $P_{\alpha}=\bar{\sigma}$ signifies the stress that would be expected in the uncoupled tissue approximation, high auxin concentration cell expansion is constrained by the remaining cells which are, in turn, under a larger amount of stress. On the other hand (bottom right) we observe that the auxin spot neighbours have, on average, negative values of $D_{\alpha}$, indicating that the largest principal stress direction is perpendicular to auxin gradients, i.e., circumferentially aligned around auxin spots, as suggested by Fig. $9 . r=0.90$ and $\eta=5.5$

Taken together, these data suggests that cell walls at the interface of a spot are under a larger amount of stress whereas the cells within auxin spots have decreased stress. This leads to reinforced polar auxin transport towards the spot and hence higher auxin concentration. The lower isotropic stress component inside the auxin spot suggests that the diffusive term inside auxin spots increases in importance relative to the active transport term.

\subsection{Tissue-wide stress coupling mitigates disruption by noise}

Up until now, our simulations were performed on hexagonal tissues in the absence of noise. This also raises the question of how tissue-wide stress patterns impact pattern emergence robustness against noise.

In plant tissue as any biological entity, noise prevails. As such cells within a tissue differ in their mechanical parameters. In order to inspect how parameter noise disrupts pattern emergence, we choose to sample reference stiffness, $E_{0}$, from a normal distribution for each cell. As outlined in the supplementary material, we expect this parameter to be the most disruptive to the active term and it is reasonable to assume it changes from cell to cell. We then simulate the resulting tissue with the uncoupled tissue approximation and tissue-wide stress coupling.

We simulate tissues with $r=0.65$ and $\eta=5.5$ for both models by promoting $E_{0}$ to a random variable sampled from Gaussian distribution with mean $\bar{E}_{0}=300 \mathrm{MPa}$ and standard deviation of $\alpha \bar{E}_{0}, \alpha \in\{0.03,0.06,0.09,0.12,0.15\}$, where $\alpha$ is the noise strength. For each value of $\alpha$, five simulations were performed per model. We fit the resulting auxin distributions 

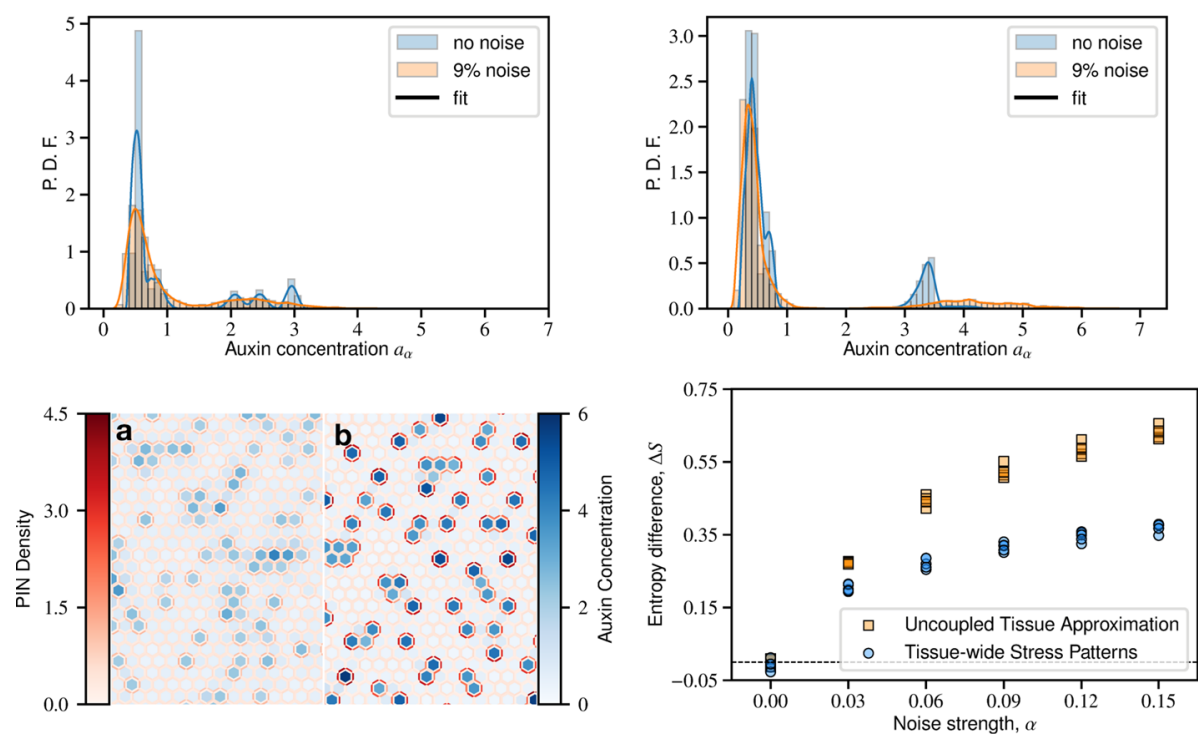

Fig. 11 Impact of noise in reference stiffness in the auxin concentration distributions for the uncoupled tissue approximation and upon tissue-wide coupling reveals robustness of auxin patterns due to tissue-wide integration. For a given noise strength, auxin concentration probability density functions (P.D.F.s) are extracted from simulation results by means of a kernel density estimation for the uncoupled tissue approximation (top left) and when considering tissue-wide stress patterns (top right). The simulated tissues have $r=0.65$ and $\eta=5.5$. For both models, we observe broadening of the distributions when considering noise. In each instance, the fit appears to be adequate for describing the resulting auxin concentration. (bottom left) Examples of the resulting patterns in the uncoupled tissue approximation (a) and in the case of tissue-wide stress patterns (b) for a noise strength of $9 \%$. Even though patterns are heavily disrupted, we can still discern more clearly high auxin concentration spikes upon tissue-wide coupling. (bottom right) Entropy difference between the resulting distributions for a given noise strength and in the absence of noise. In the presence of tissue-wide stress patterns disruption of tissue patterning is consistently lower than in the uncoupled tissue approximation

to a probability density function (Fig. 11 top left and top right). We observe that noise in reference stiffness impacts the patterning behaviour in a severe manner (Fig. 11 bottom left). Yet, with tissue-wide stress coupling spots of noticeable auxin accumulation are preserved.

In order to quantify the disruption, we compute the entropy (Eq. 20) of a fitted auxin probability density function by means of a kernel density estimation on the resulting auxin distributions. The kernel used for all fits was the Epanechnikov kernel with a bandwidth of about 0.202 . This number arises in the rule-of-thumb estimate for the Gaussian kernel for the sample size and dimension of this system. The partition size used for the numerical approximation of the entropy is the same for all instances. Afterwards, we measure the entropy difference between the expected auxin distribution for each value of $\alpha$, of each model and for all simulations (Fig. 11 bottom right). The reference entropy is taken to be the average of the uncoupled tissue approximation at $\alpha=0$. We can infer from these results that tissue-wide coupling helps to rescue auxin accumulation spots despite its heavy disruption in comparison to the uncoupled tissue approximation.

\subsection{High turgor preferred but not required for sustaining auxin maxima}

It is of interest to the experimental community at this point in time how auxin spots and turgor pressure correlates $[63,64]$. To explore how the the tissue-wide mechanical model 


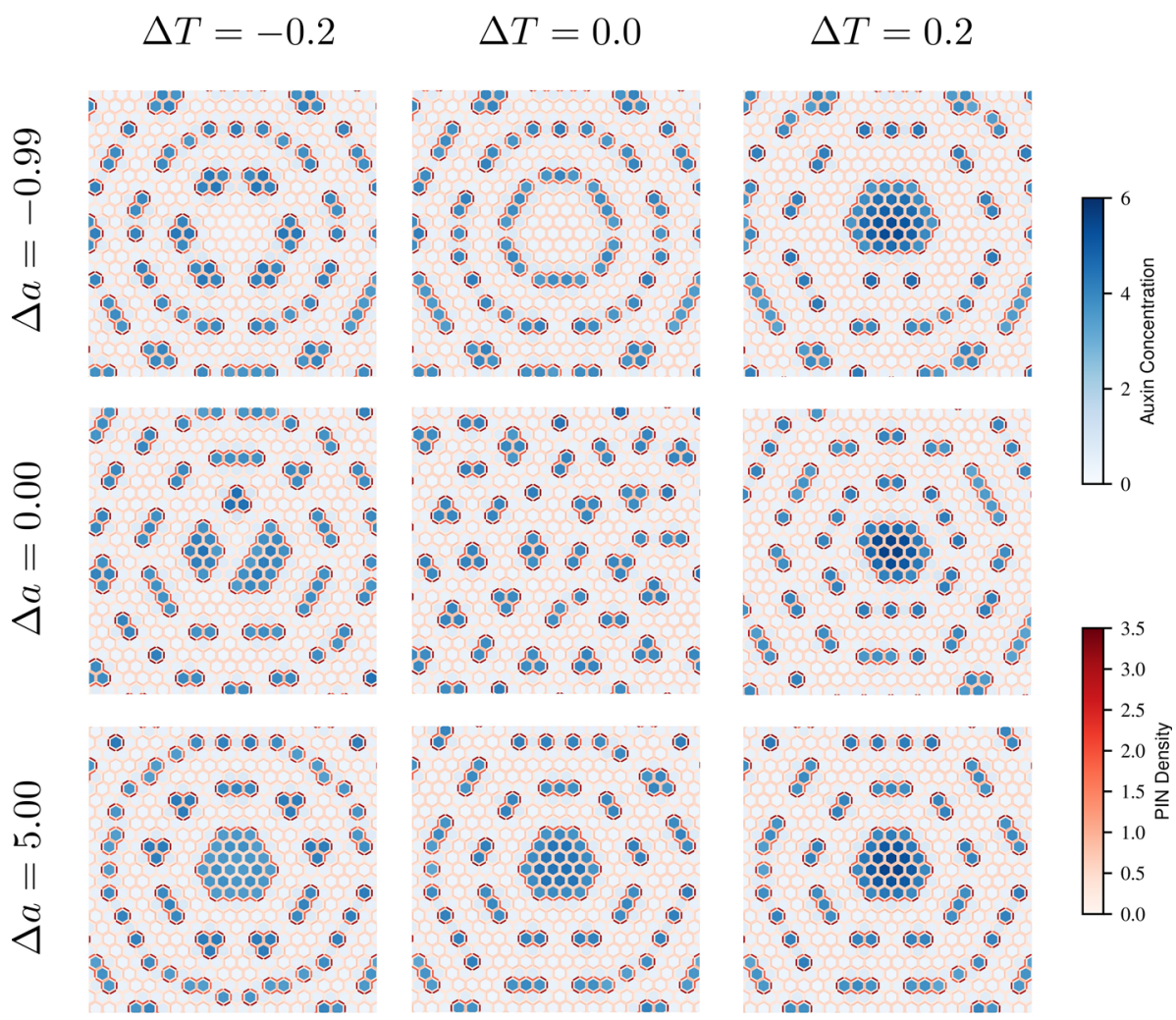

Fig. 12 Simulations of auxin patterning with the tissue-wide mechanical model when considering a local turgor increase (right column), decrease (left column), or constant (middle column), and a prior high (bottom row), low (top row), or constant (middle row) initial auxin concentration. Units of $\Delta T$ are MPa. We used $\eta=10$ and $r=0.65$ for all simulations. Even if high turgor predicts an auxin maximum, it becomes unclear what might happen with low turgor. The tissue-wide mechanical model seems to preserve already existing auxin maxima

responds to local turgor variations, we probe what happens when patterns emerge with a local increase or decrease in turgor. Since stress is tied to active auxin flow, the results are prone to be affected by prior auxin concentration distribution. Hence, we test the several turgor scenarios as well as initial auxin concentration. We added a contribution to turgor of $\Delta T e^{-\frac{x^{2}+y^{2}}{2 \sigma}}$, where $\Delta T \in\{-0.2,0.0,0.2\} \mathrm{MPa}$ and $\sigma=2 L$. For initial auxin concentration, we used the same form with the same $\sigma$, yet the largest deviations are $\Delta a \in\{-0.99,0.00,5.00\}$. To be sure we are well within the pattern formation regime of the model for low pressure, we used the stress-PIN coupling value of $\eta=10$ and $r=0.65$.

Regardless of initial auxin concentration, for high turgor, we observe an auxin maximum predictably emerges correlated with a turgor maximum (Fig. 12 right column). Nevertheless, if an initial auxin concentration exists, we also predict that the spot remains regardless of whether this position is a turgor minimum or not (Fig. 12 bottom row). Low turgor regions can still exhibit patterns adding to the complexity of this simple measure (Fig. 12 middle row, left).

From these data, we can conclude is that developmental history is as important as turgor pressure for predicting auxin maxima positioning. We can predict high turgor leads to auxin 
accumulation, yet low turgor gives us little insight on auxin distribution. We can also predict that a high auxin concentration region.

\section{Discussion}

Here, we used a model composed of a vertex model for plant tissue mechanics, and a compartment model for auxin transport to uncover the role of tissue-wide mechanical coupling on auxin redistribution. We first verified that the tissue-wide mechanical model successfully captures the behaviour of plant tissue upon ablation experiments and the conditions for emergence of auxin patterns. We then compared the behaviour of our model featuring tissue-wide mechanical coupling to an approximation which regards cells as mechanically isolated. We observe the emergence of focused auxin spots with high auxin concentration when tissuewide mechanical coupling is implemented. Notably, depending on the parameters of the tissue-wide stress model, auxin spot concentration is more sensitive to stress than what could be predicted from the approximation. We observe that tissue-wide mechanical effects unaccounted for by the approximation have a positive impact on PIN polarity. Furthermore, we show that stress patterning of the tissue mitigates the disruption caused by noise, increasing robustness of the system. Finally, we observe auxin concentration correlating with high local turgor pressure. This behaviour coexists with the possibility of having auxin maxima anti-correlating with turgor.

The auxin-induced cell wall loosening effect ( $r$ parameter in this work) is an important determinant of the feedback of auxin on tissue mechanics. The range of values of $r$ for which substantial pattern focusing occurs is around $r \sim 0.60$ and above in our model. This translates into a variation of stiffness from a minimum value $E_{\min }$ up to $E_{\max }=4 E_{\min }$ (see supplementary material). Although high, this range is within biological expectation and supported by AFM measurements on auxin treated tissues [7] and comparable to previous simulations of this mechanism [10] where $E_{\max } / E_{\min }=5$ which translates into $r=2 / 3$.

Comparison of the tissue-wide stress patterning case to the uncoupled tissue approximation reveals that auxin spot concentration has a very steep transition in the former case (Fig. 8). This results in a several-fold increase in auxin concentration at values of $\eta$ close to the threshold for pattern formation. What was once a relatively subtle graded response of auxin spot concentration on stress behaves now as an on-off switch by virtue of tissue mechanical relaxation. Since the mechanical perturbations being highlighted through the comparison are purely passive, this improvement in sensing comes at no additional cost for the plant and therefore has the potential to increase efficiency.

In the present work, we explored the parameter space $(\eta, r)$ exclusively. We observe consistently that pattern wavelengths shorten from the uncoupled tissue approximation and the tissue-wide coupling model. It would be interesting to systematically probe the diversity of patterns and how they change upon tissue-wide mechanical coupling. For our simulations, we used the parameters $n, m, K$ from [10], parameters on which we have little empirical information. Yet, the sensitivity analysis from [29] suggests that $n$ and $K$ especially should affect patterning the most. We speculate the parameter $m$, specific to wall loosening, to be of similar importance. We expect that a study focusing on these three parameters would yield more interesting patterns shapes.

This work focused exclusively in the hypothesis that PIN is mechanically regulated. However, competing chemical feedback mechanisms have been proposed. Recently, mechanics and ARF-mediated PIN expression have been modelled together by [40] and show promising pattern formation capabilities. Other factors we have not taken into account is the family of 
auxin importers of the AUX family, which have been shown to be present in the epidermal layer of the shoot apical meristem [16]. Auxin binding proteins have also been hypothesized to promote auxin flow polarization [65]. Another observed interaction is cytokinin action controlling PIN polarity during lateral root formation [66].

The PIN regulation used in the auxin transport compartment model was specifically stressbased. In the supplementary text, we show results using strain-based PIN binding instead. We observe the same overall auxin spot focusing behaviour. It is still unclear whether the PIN density change due to mechanics is a result of strain or stress [11]. In fact, this question has been tackled recently by [54] concluding that in most simulated experiments both strain and stress-based models behave similarly. A notable exception is the experimentally observed correlation of PIN polarity and auxin concentration [37,67]. On one hand, this observation is not captured by the stress-based PIN binding model. On the other hand, available experimental data and simulations suggest stress sensing being easier to explain [54]. Furthermore, the polarity difference could be rescued by the observation that ARF-mediated PIN expression is higher at the tip of the primordium [68].

The specific distribution of emergent stress patterns is remarkable in the sense that it coincides with the shape-induced stress patterns, as indicated by microtubule orientation, around the tip of the primordium as it emerges from the meristem [41]. Therefore, tissue-wide stress patterning sets the stage for primordium outgrowth by focusing efficiently auxin, forming local circumferential stress that in turn may re-orient microtubules and prefigure the shape of the primordium. This process could, in turn, be capable of reinforcing auxin transport to the tip of the newly forming organ. Yet, quantifying this requires further modelling. Therefore, it would be interesting to include auxin transport in already existing models for primordium outgrowth $[8,9]$.

Even though the analysed numerical simulations were limited to noise in the parameter $E_{0}$, it showcases the power of the aforementioned auxin peak focusing that happens upon tissue-wide mechanical coupling. In this instance, we show here the power of tissue-wide stress patterns to mitigate the information loss due to noise by inspecting entropy of auxin concentration distribution. This result, especially when paired with the increase in sensitivity mentioned above, is indeed remarkable. This is due to the fact that in a wide range of optimized systems, biological or otherwise, robustness and efficiency are thought to be in opposition to each other, as illustrated, for example, by [69]. This is because robustness is usually brought upon by additional systems which would be considered clutter by a system geared towards efficiency. This opens a novel line of argumentation in the discourse of the evolution of mechanical signalling in multicellular organisms.

Lastly, we probed the behaviour of the used tissue-wide mechanical model when faced with local turgor variations. Our results indicate that once established auxin spots can endure low turgor scenarios, even if they would prefer high turgor regions all else being equal. Maintaining a turgor pressure difference for so long, however, might not be feasible for the plant. To answer how this setting could be achieved would require modelling water transport between plant cells along the lines of [63]. Nevertheless, our model can explain, at least in part, why these two quantities do not correlate in a straightforward manner.

\section{Conclusion}

Even though the mechanisms by which PIN preferentially associate with stressed cell walls is unclear, here we show that there are substantial advantages by intertwining tissue-wide mechanics and auxin patterning. Even if auxin patterning is possible by chemical processes 
and local mechanical coupling, tissue-wide mechanics may provide a way for patterning to still occur at a lower energy cost for the tissue. Moreover, this process can also provide robustness to the patterning, factoring in tissue-wide stress pattern, a sort of proprioceptive mechanism.

Acknowledgements This work was supported by the Max Planck Society and the Deutsche Forschungsgemeinschaft via DFG-FOR2581.

Author contributions JRDR, AM and KA designed research. JRDR performed the research. JRDR, AM and KA wrote the article.

Funding This work has been Funded by Deutsche Forschungsgemeinschaft via FOR-2581 (P1, P6).

Data availibility The data that support the findings of this study are available from the corresponding author upon request.

\section{Compliance with ethical standards}

Conflict of interest The authors decalre that they have no conflict of interest.

Code availibility The code used to produce the data of this study are available from the corresponding author upon request.

Open Access This article is licensed under a Creative Commons Attribution 4.0 International License, which permits use, sharing, adaptation, distribution and reproduction in any medium or format, as long as you give appropriate credit to the original author(s) and the source, provide a link to the Creative Commons licence, and indicate if changes were made. The images or other third party material in this article are included in the article's Creative Commons licence, unless indicated otherwise in a credit line to the material. If material is not included in the article's Creative Commons licence and your intended use is not permitted by statutory regulation or exceeds the permitted use, you will need to obtain permission directly from the copyright holder. To view a copy of this licence, visit http://creativecommons.org/licenses/by/4.0/.

\section{References}

1. J.A. Lockhart, An analysis of irreversible plant cell elongation. J. Theor. Biol. 8, 264-275 (1965)

2. J.K.E. Ortega, Augmented growth equation for cell wall expansion. Plant Physiol. 79, 318-320 (1985)

3. D. Cosgrove, Biophysical control of plant cell growth. Ann. Rev. Plant Physiol. 37, 377-405 (1986)

4. A. Geitmann, J.K. Ortega, Mechanics and modeling of plant cell growth. Trends Plant Sci. 14, 467-478 (2009)

5. O. Hamant, J. Traas, The mechanics behind plant development. New Phytol. 185, 369-385 (2010)

6. A. Peaucelle, S.A. Braybrook, L. Le Guillou, E. Bron, C. Kuhlemeier, H. Höfte, Pectin-induced changes in cell wall mechanics underlie organ initiation in arabidopsis. Curr. Biol. 21, 1720-1726 (2011)

7. S.A. Braybrook, A. Peaucelle, Mechano-chemical aspects of organ formation in Arabidopsis thaliana: the relationship between auxin and pectin. PLoS ONE 8, e57813 (2013)

8. F. Boudon, J. Chopard, O. Ali, B. Gilles, O. Hamant, A. Boudaoud, J. Traas, C. Godin, A computational framework for 3D mechanical modeling of plant morphogenesis with cellular resolution. PLoS Comput. Biol. 11, e1003950 (2015)

9. J. Khadka, J.-D. Julien, K. Alim, Feedback from tissue mechanics self-organizes efficient outgrowth of plant organ. Biophys. J . 117, 1995-2004 (2019)

10. M.G. Heisler, O. Hamant, P. Krupinski, M. Uyttewaal, C. Ohno, H. Jönsson, J. Traas, E.M. Meyerowitz, Alignment between PIN1 polarity and microtubule orientation in the shoot apical meristem reveals a tight coupling between morphogenesis and auxin transport. PLoS Biol. 8, e1000516 (2010)

11. N. Nakayama, R.S. Smith, T. Mandel, S. Robinson, S. Kimura, A. Boudaoud, C. Kuhlemeier, Mechanical regulation of auxin-mediated growth. Curr. Biol. 22, 1468-1476 (2012)

12. W.D. Teale, I.A. Paponov, K. Palme, Auxin in action: signalling, transport and the control of plant growth and development. Nat. Rev. Mol. Cell Biol. 7, 847-859 (2006)

13. S. Vanneste, J. Friml, Auxin: a trigger for change in plant development. Cell 136, 1005-1016 (2009) 
14. M. Sassi, T. Vernoux, Auxin and self-organization at the shoot apical meristem. J. Exp. Bot. 64, 2579-2592 (2013)

15. E. Benková, M. Michniewicz, M. Sauer, T. Teichmann, D. Seifertová, G. Jürgens, J. Friml, Local, effluxdependent auxin gradients as a common module for plant organ formation. Cell 115, 591-602 (2003)

16. D. Reinhardt, E.-R. Pesce, P. Stieger, T. Mandel, K. Baltensperger, M. Bennett, J. Traas, J. Friml, C. Kuhlemeier, Regulation of phyllotaxis by polar auxin transport. Nature 426(6964), 255-60 (2003)

17. P.B. de Reuille, I. Bohn-Courseau, K. Ljung, H. Morin, N. Carraro, C. Godin, J. Traas, Computer simulations reveal properties of the cell-cell signaling network at the shoot apex in Arabidopsis. Proc. Nat. Acad. Sci. 103, 1627-1632 (2006)

18. D. Reinhardt, T. Mandel, C. Kuhlemeier, Auxin regulates the initiation and radial position of plant lateral organs. Plant Cell 12, 507 (2000)

19. R.S. Smith, The role of auxin transport in plant patterning mechanisms. PLoS Biol. 6, e323 (2008)

20. T. Vernoux, F. Besnard, J. Traas, Auxin at the shoot apical meristem. Cold Spring Harb. Perspect. Biol. 2, a001487-a001487 (2010)

21. S. Robinson, A. Burian, E. Couturier, B. Landrein, M. Louveaux, E.D. Neumann, A. Peaucelle, A. Weber, N. Nakayama, Mechanical control of morphogenesis at the shoot apex. J. Exp. Bot. 64, 4729-4744 (2013)

22. J. Petrasek, PIN proteins perform a rate-limiting function in cellular auxin efflux. Science 312, 914-918 (2006)

23. J. Wisniewska, Polar PIN localization directs auxin flow in plants. Science 312, 883-883 (2006)

24. J. Petrasek, J. Friml, Auxin transport routes in plant development. Development 136, 2675-2688 (2009)

25. H. Jonsson, M.G. Heisler, B.E. Shapiro, E.M. Meyerowitz, E. Mjolsness, An auxin-driven polarized transport model for phyllotaxis. Proc. Nat. Acad. Sci. 103, 1633-1638 (2006)

26. R.S. Smith, S. Guyomarc'h, T. Mandel, D. Reinhardt, C. Kuhlemeier, P. Prusinkiewicz, A plausible model of phyllotaxis. Proc. Nat. Acad. Sci. 103, 1301-1306 (2006)

27. R.M. Merks, Y. Van de Peer, D. Inzé, G.T. Beemster, Canalization without flux sensors: a traveling-wave hypothesis. Trends Plant Sci. 12, 384-390 (2007)

28. A.C. Newell, P.D. Shipman, Z. Sun, Phyllotaxis: cooperation and competition between mechanical and biochemical processes. J. Theor. Biol. 251, 421-439 (2008)

29. P. Sahlin, B. Söderberg, H. Jönsson, Regulated transport as a mechanism for pattern generation: capabilities for phyllotaxis and beyond. J. Theor. Biol. 258, 60-70 (2009)

30. T. Sachs, Polarity and the induction of organized vascular tissues. Ann. Bot. 33, 263-275 (1969)

31. G.J. Mitchison, A model for vein formation in higher plants, in Proceedings of the Royal Society of London. Series B. Biological Sciences, vol. 207, pp. 79-109 (1980)

32. F.G. Feugier, A. Mochizuki, Y. Iwasa, Self-organization of the vascular system in plant leaves: interdependent dynamics of auxin flux and carrier proteins. J. Theor. Biol. 236, 366-375 (2005)

33. F.G. Feugier, Y. Iwasa, How canalization can make loops: a new model of reticulated leaf vascular pattern formation. J. Theor. Biol. 243, 235-244 (2006)

34. H. Fujita, A. Mochizuki, Pattern formation of leaf veins by the positive feedback regulation between auxin flow and auxin efflux carrier. J. Theor. Biol. 241, 541-551 (2006)

35. S. Stoma, M. Lucas, J. Chopard, M. Schaedel, J. Traas, C. Godin, Flux-based transport enhancement as a plausible unifying mechanism for auxin transport in meristem development. PLoS Comput. Biol. 4, e1000207 (2008)

36. K. Alim, E. Frey, Quantitative predictions on auxin-induced polar distribution of PIN proteins during vein formation in leaves. Eur. Phys. J. E 33, 165-173 (2010)

37. E.M. Bayer, R.S. Smith, T. Mandel, N. Nakayama, M. Sauer, P. Prusinkiewicz, C. Kuhlemeier, Integration of transport-based models for phyllotaxis and midvein formation. Genes Dev. 23, 373-384 (2009)

38. M. Cieslak, A. Runions, P. Prusinkiewicz, Auxin-driven patterning with unidirectional fluxes. J. Exp. Bot. 66, 5083-5102 (2015)

39. Y. Hayakawa, M. Tachikawa, A. Mochizuki, Mathematical study for the mechanism of vascular and spot patterns by auxin and pin dynamics in plant development. J. Theor. Biol. 365, 12-22 (2015)

40. H.R. Allen, M. Ptashnyk, Mathematical modelling of auxin transport in plant tissues: flux meets signalling and growth. Bull. Math. Biol. 82, 1-35 (2020)

41. O. Hamant, M.G. Heisler, H. Jonsson, P. Krupinski, M. Uyttewaal, P. Bokov, F. Corson, P. Sahlin, A. Boudaoud, E.M. Meyerowitz, Y. Couder, J. Traas, Developmental patterning by mechanical signals in arabidopsis. Science 322, 1650-1655 (2008)

42. O. Hamant, E.S. Haswell, Life behind the wall: sensing mechanical cues in plants. BMC Biol. 15, 59 (2017)

43. O. Hamant, D. Inoue, D. Bouchez, J. Dumais, E. Mjolsness, Are microtubules tension sensors? Nat. Commun. 10, 2360 (2019) 
44. M. Uyttewaal, A. Burian, K. Alim, B. Landrein, D. Borowska-Wykręt, A. Dedieu, A. Peaucelle, M. Ludynia, J. Traas, A. Boudaoud, D. Kwiatkowska, O. Hamant, Mechanical stress acts via katanin to amplify differences in growth rate between adjacent cells in arabidopsis. Cell 149, 439-451 (2012)

45. E. Feraru, M.I. Feraru, J. Kleine-Vehn, A. Martinière, G. Mouille, S. Vanneste, S. Vernhettes, J. Runions, J. Friml, PIN polarity maintenance by the cell wall in arabidopsis. Curr. Biol. 21, 338-343 (2011)

46. H. Li, D. Lin, P. Dhonukshe, S. Nagawa, D. Chen, J. Friml, B. Scheres, H. Guo, Z. Yang, Phosphorylation switch modulates the interdigitated pattern of PIN1 localization and cell expansion in Arabidopsis leaf epidermis, in Cell Research, vol. 21, pp. 970-978 (2011)

47. P.J. Overvoorde, Functional genomic analysis of the AUXIN/INDOLE-3-ACETIC ACID gene family members in Arabidopsis thaliana. Plant Cell Online 17, 3282-3300 (2005)

48. D.B. Staple, R. Farhadifar, J.C. Röper, B. Aigouy, S. Eaton, F. Jülicher, Mechanics and remodelling of cell packings in epithelia. Eur. Phys. J. E 33, 117-127 (2010)

49. Y. Li, H. Naveed, S. Kachalo, L.X. Xu, J. Liang, Mechanisms of regulating cell topology in proliferating epithelia: impact of division plane, mechanical forces, and cell memory. PLoS ONE 7, e43108 (2012)

50. G. Trichas, A.M. Smith, N. White, V. Wilkins, T. Watanabe, A. Moore, B. Joyce, J. Sugnaseelan, T.A. Rodriguez, D. Kay, R.E. Baker, P.K. Maini, S. Srinivas, Multi-cellular rosettes in the mouse visceral endoderm facilitate the ordered migration of anterior visceral endoderm cells. PLoS Biol. 10, e1001256 (2012)

51. N. Murisic, V. Hakim, I.G. Kevrekidis, S.Y. Shvartsman, B. Audoly, From discrete to continuum models of three-dimensional deformations in epithelial sheets. Biophys. J . 109, 154-163 (2015)

52. M. Osterfield, X. Du, T. Schüpbach, E. Wieschaus, S.Y. Shvartsman, Three-dimensional epithelial morphogenesis in the developing drosophila egg. Dev. Cell 24, 400-410 (2013)

53. S. Alt, P. Ganguly, G. Salbreux, Vertex models: from cell mechanics to tissue morphogenesis. Philos. Trans. R. Soc. B Biol. Sci. 372, 20150520 (2017)

54. J.-D. Julien, A. Pumir, A. Boudaoud, Strain- or stress-sensing in mechanochemical patterning by the phytohormone auxin. bioRxiv 81, 3342-3361 (2019)

55. L. Guibas, J. Stolfi, Primitives for the manipulation of general subdivisions and the computation of voronoi. ACM Trans. Graph. 4, 74-123 (1985)

56. P. Heckbert, Quad-edge data structure library. http://www.cs.cmu.edu/afs/andrew/scs/cs/15-463/2001/ $\mathrm{pub} / \mathrm{src} / \mathrm{a} 2 / \mathrm{cell} /$

57. J. Nocedal, Updating quasi-Newton matrices with limited storage. Math. Comput. 35, 773 (1980)

58. D.C. Liu, J. Nocedal, On the limited memory BFGS method for large scale optimization. Math. Program. 45, 503-528 (1989)

59. S.G. Johnson, The nlopt nonlinear-optimization package. http://github.com/stevengj/nlopt

60. M. Galassi et al., Gnu Scientific Library Reference Manual, 3rd edn. (Network Theory Ltd., Surrey, 2009).

61. S. Weglarczyk, Kernel density estimation and its application. ITM Web Conf. 23, 00037 (2018)

62. O. Leyser, Auxin signaling. Plant Physiol. 176, 465-479 (2018)

63. Y. Long, I. Cheddadi, G. Mosca, V. Mirabet, M. Dumond, A. Kiss, J. Traas, C. Godin, A. Boudaoud, Cellular heterogeneity in pressure and growth emerges from tissue topology and geometry. Curr. Biol. 30(8), 1504-1516.e8 (2020)

64. I. Cheddadi, M. Génard, N. Bertin, C. Godin, Coupling water fluxes with cell wall mechanics in a multicellular model of plant development. PLOS Comput. Biol. 15, 1-16 (2019)

65. M. Adamowski, J. Friml, PIN-dependent auxin transport: action, regulation, and evolution. Plant Cell Online 27, 20-32 (2015)

66. P. Marhavý, J. Duclercq, B. Weller, E. Feraru, A. Bielach, R. Offringa, J. Friml, C. Schwechheimer, A. Murphy, E. Benková, Cytokinin controls polarity of PIN1-dependent auxin transport during lateral root organogenesis. Curr. Biol. 24, 1031-1037 (2014)

67. D.L. O'Connor, A. Runions, A. Sluis, J. Bragg, J.P. Vogel, P. Prusinkiewicz, S. Hake, A division in pin-mediated auxin patterning during organ initiation in grasses. PLOS Comput. Biol. 10, 1-14 (2014)

68. M.G. Heisler, C. Ohno, P. Das, P. Sieber, G.V. Reddy, J.A. Long, E.M. Meyerowitz, Patterns of auxin transport and gene expression during primordium development revealed by live imaging of the arabidopsis inflorescence meristem. Curr. Biol. 15, 1899-1911 (2005)

69. G. Peng, S.-Y. Tan, J. Wu, P. Holme, Trade-offs between robustness and small-world effect in complex networks. Sci. Rep. 6, 37317 (2016) 